\title{
Tuning of Spectral Sensitivity of AlGaN/GaN UV Detector
}

\author{
K.P. Korona*, A. Drabińska, A. Trajnerowicz, R. Bożek, \\ K. PAKUŁa AND J.M. Baranowski \\ Institute of Experimental Physics, Warsaw University \\ Hoża 69, 00-681 Warsaw, Poland
}

\begin{abstract}
GaN/AlGaN photodetector that exhibits new interesting property is presented. Its spectral sensitivity depends upon bias voltage. Under positive or low negative bias the detector is sensitive mainly to the ultrafiolet radiation absorbed by AlGaN layer $3.7-3.8 \mathrm{eV}$. Under negative bias $U_{\mathrm{B}}$ below $-4 \mathrm{~V}$, the detector is sensitive mainly to the radiation absorbed by $\mathrm{GaN}$ $(3.4-3.6 \mathrm{eV})$. The effect can be explained based on numerical calculations of the electric field and potential profiles of this structure. The damping of GaN signal is attributed to activity of $2 \mathrm{D}$ electron gas formed on the GaN/AlGaN interface by spontaneous polarization. The reappearing of the signal is attributed to tunneling of holes through AlGaN, stimulated by a high electric field.
\end{abstract}

PACS numbers: 73.40.Kp, 73.50.Pz, 78.40.Fy

\section{Introduction}

Considering current advances in nitride technology, AlGaN/GaN structure is the most promising material for photodetection in the ultraviolet (UV) region of the spectrum, suitable to develop efficient visible-blind [1] and solar-blind sensors [2]. Taking into account its superior radiation hardness and high temperature resistance, this material is suitable for devices working in extreme conditions. Applications range from space communications to ozone layer monitoring or flame detection.

Up to now, many promising $\mathrm{AlGaN} / \mathrm{GaN}$ detector structures have been studied [2-5]. The results show that AlGaN/GaN, $p-i-n$ heterodiodes show considerable promise as solar-blind photodetectors [2]. On the other hand, the Schottky

*corresponding author; e-mail: kkorona@fuw.edu.pl 
barrier detectors based on $\mathrm{Al}_{0.22} \mathrm{Ga}_{0.28} \mathrm{~N}$ having a visible rejection of 3 to 4 orders of magnitude proved to be very fast. A minimum decay time as short as 15 ns was reported for these diodes [4]. Furthermore, it has been found [5] that high charge density, two-dimensional (2D) electron gas can be realized even without any doping, caused by the strong piezoelectric and spontaneous polarization effects natural to the hexagonal group-III nitrides [6]. Interesting effects can be obtained by introducing ultrathin AlN interlayer producing 2D electron gas inside GaN [7].

Here we present a detector structure consisting of two active layers: undoped $\mathrm{Al}_{x} \mathrm{Ga}_{1-x} \mathrm{~N}$ and undoped GaN, both partially strained. This structure contains 2D electron gas at the GaN/AlGaN interface that can be controlled by external bias. The increase or decrease in electron concentration leads to changes in minority carriers lifetime and distribution of the electric field. In this way, it is possible to control spectral sensitivity of the detector. This property can be used, in detectors that can simultaneously measure UV A and UV B radiation and distinguish them.

\section{Experiment}

The structure was grown by metalorganic chemical vapor deposition (MOCVD) on the sapphire substrate. Two active layers of undoped $\mathrm{Al}_{x} \mathrm{Ga}_{1-x} \mathrm{~N}$ ( $x=0.11$ and $0.17, d=60$ and $70 \mathrm{~nm})$ and undoped $\mathrm{GaN}(d=140 \mathrm{~nm})$, both nearly relaxed were grown on a thick conductive GaN:Si layer. The nominal donor concentration of the GaN:Si layer was $N_{\mathrm{D}}=2.0 \times 10^{18} \mathrm{~cm}^{-3}$. A very thin GaN cap layer was grown on top to protect AlGaN layer. Semitransparent, gold, Schottky contacts were evaporated on the surface. The ohmic contacts were alloyed to make connection to the GaN:Si layer (see Fig. 1).

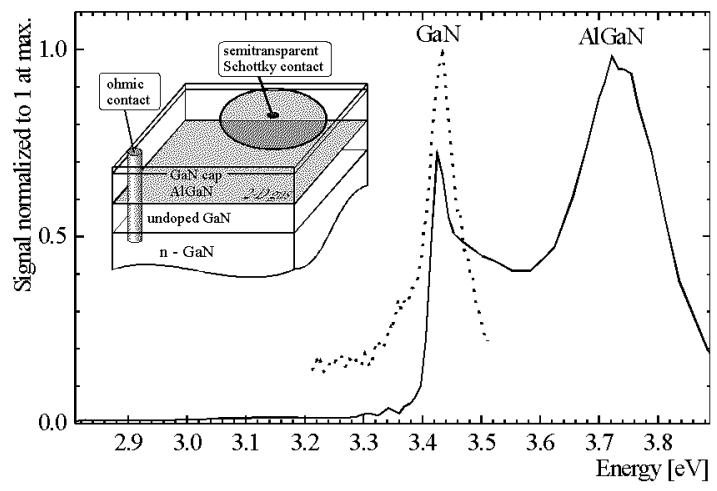

Fig. 1. Photoluminescence (dotted line) and photocurrent at $U_{\mathrm{B}}=-4 \mathrm{~V}$ (solid line) spectra of the $\mathrm{Al}_{0.11} \mathrm{Ga}_{0.89} \mathrm{~N} / \mathrm{GaN}$ photodetector (structure scheme in the inset) at room temperature. 
The samples showed photoluminescence, visible even at room temperature (see Fig. 2). The photocurrent (PC) spectra were measured in temperature range from 8 to $300 \mathrm{~K}$, under different bias voltages. Illumination intensity was $0.1-1 \mu \mathrm{W} / \mathrm{cm}^{2}$.

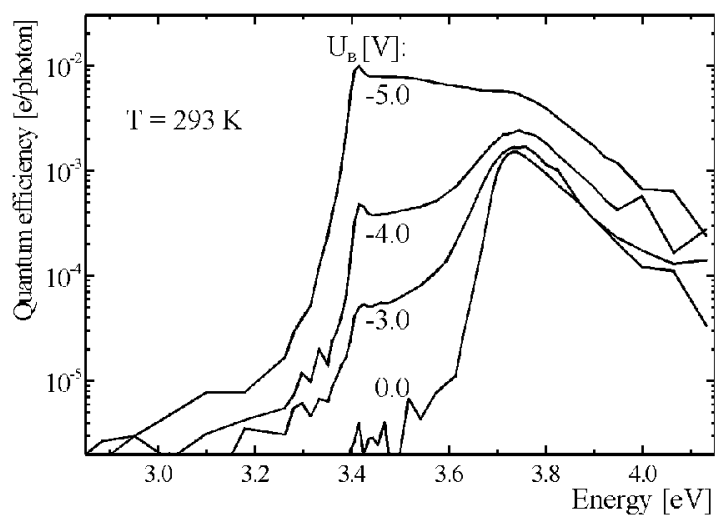

Fig. 2. Bias-dependent spectra of the $\mathrm{AlGaN} / \mathrm{GaN}$ photodetector. Signal with maximum at $3.73 \mathrm{eV}$ is due to $\mathrm{AlGaN}$, the maximum at $3.42 \mathrm{eV}$ is due to $\mathrm{GaN}$.

It was observed that the $\mathrm{PC}$ spectrum depended upon bias voltage $\left(U_{\mathrm{B}}\right)$. Under positive or low negative bias the detector was sensitive mainly to UV radiation absorbed by AlGaN layer (see Fig. 2). The signal from $\mathrm{Al}_{x} \mathrm{Ga}_{1-x} \mathrm{~N}$ depended upon $\mathrm{Al}$ content. For example, its maxima were at $3.73 \mathrm{eV}$ and $3.84 \mathrm{eV}$ for $x=0.11$ and 0.17 , respectively. At $U_{\mathrm{B}}=0 \mathrm{~V}$, the sensitivity for radiation below the AlGaN threshold was at least two orders of magnitude lower than the sensitivity at the maximum. Application of negative voltage led to an increase in the sensitivity of $\mathrm{GaN}$. At room temperature the signal from $\mathrm{GaN}$ started at about $3.4 \mathrm{eV}$ and reached maximum at the energy of $3.42 \mathrm{eV}$. At about $-4 \mathrm{~V}$ the signals form GaN and AlGaN had similar amplitudes. Spectra measured at $U_{\mathrm{B}}=-5 \mathrm{~V}$ and below were dominated by photocurrent related to the excitation of GaN. The effect was fully reversible, after return to zero bias the sensitivity in the GaN spectral range decreased.

The dramatic change of the GaN-related sensitivity in the range 3.4-3.6 eV can be described by the ratio of $\mathrm{PC}$ at $U_{\mathrm{B}}=-5 \mathrm{~V}$ to $\mathrm{PC}$ at $0 \mathrm{~V}$, which was equal to about 2500 (at $3.4 \mathrm{eV}$ ). A slight increase in $\mathrm{PC}$ at $3.7 \mathrm{eV}$ can be explained by the increase in the signal from $\mathrm{GaN}$, which was also sensitive for photons of $3.7 \mathrm{eV}$ energy. The effect had a weak thermal dependence. The decrease in temperature caused a parallel decrease in sensitivity of GaN and AlGaN layers. The maxima of sensitivity moved to higher energies in agreement with the expected increase in the band gap.

Electroreflectance bias-wavelength (ERBW) mapping technique [8] was used to analyze electric field in the structure. A Schottky diode biased with a sum of 
DC voltage $(U)$ and AC modulation signal $\left(U_{0} \cos \omega t\right)$ was illuminated with a monochromatic light of wavelength $\lambda$. Reflected light was measured with a voltmeter and lock-in analyzer simultaneously. The signals measured for various biases and wavelengths formed the electroreflectance (ER) maps. In wavelength domain, clear Franz-Keldysh oscillations (FKOs) were observed.

The FKOs can be described using asymptotic expansions for Airy functions [9]:

$$
\frac{\Delta R}{R} \propto \frac{1}{E^{2}\left(E-E_{\mathrm{g}}\right)} \exp \left[\frac{-2\left(E-E_{\mathrm{g}}\right)^{1 / 2} \Gamma}{(\hbar \Omega)^{3 / 2}}\right] \cos \left[\frac{4}{3} \frac{\left(E-E_{\mathrm{g}}\right)^{3 / 2}}{(\hbar \Omega)^{3 / 2}}+\varphi\right],
$$

where $E_{\mathrm{g}}$ is an energy gap, $\varphi-$ a phase factor, $\Gamma$ - a broadening parameter, and $\hbar \Omega$ is an electro-optical energy, connected with reduced effective mass of electron-hole pair $\left(\mu_{\|}\right)$and an electric field $(F)$ by the expression $(\hbar \Omega)^{3}=$ $(e h F)^{2} / 2 \mu_{\|}$. Due to a periodic dependence of the $\Delta R / R$ signal as a function of $\left(E-E_{\mathrm{g}}\right)^{3 / 2}$ it is possible to calculate the electric field using the Fourier transform (FT) analysis.

Based on the ERBW technique, a high electric field of the order of $500 \mathrm{kV} / \mathrm{cm}$ was found in the AlGaN layer. The field was obviously caused by spontaneous polarization of the GaN/AlGaN interface [7]. The electric field in the GaN layer was very low (below $20 \mathrm{kV} / \mathrm{cm}$ ) at positive or low negative biases. The field in the $\mathrm{GaN}$ layer reached measurable values at bias below $-2 \mathrm{~V}$. At $U_{\mathrm{B}}=-4 \mathrm{~V}$, it was about $100 \mathrm{kV} / \mathrm{cm}$.

\section{Numerical calculations}

The experimental results can be compared with theoretical expectations based on the simple numerical model. Electrical charge is a source of electrical field that changes the potential along the structure. On the other hand, concentration of the charged particles depends upon potential, so the charge, field, and potential distributions $(n(z), p(z), E(z)$, and $V(z))$ must be calculated simultaneously by a self-consistent procedure $[10,11]$. Since the sample is under illumination, the Fermi level is not constant along the sample, so the Fermi-Dirac distribution cannot be used directly to determine the carrier concentration. Instead, it is determined by solving electrical transport equations involving drift in an electric field, diffusion, generation by light, and recombination.

The electric field $E(z)$ is generated by the bias $U$ applied to the sample, by electrical charges $\rho(z)$ (electrons $n$, holes $p$, ionized donors $n_{\mathrm{D}}^{+}$and acceptors $n_{\mathrm{A}}^{-}$). Since, the III-V nitrides possess a large spontaneous and piezoelectric polarization [7]. Therefore, it is necessary to include also the polarization on the GaN/AlGaN interface $P_{\mathrm{I}}$ :

$$
E(z, t)=\frac{U}{d}+\frac{1}{\varepsilon \varepsilon_{0}}\left\{P_{\mathrm{I}}+e \int_{0}^{z}\left[-n\left(z^{\prime}\right)+p\left(z^{\prime}\right)+n_{\mathrm{D}}^{+}\left(z^{\prime}\right)-n_{\mathrm{A}}^{-}\left(z^{\prime}\right)\right] \mathrm{d} z^{\prime}\right\},
$$

where $d$ is the effective thickness of the structure. 
The results of the numerical calculations are presented in Fig. 3. It has been found that the Schottky barrier and polarization at GaN/AlGaN interface $P_{\mathrm{I}}$ cause a strong electric field present in AlGaN layer, in agreement with the electroreflectance measurements (reported in the previous section). Since layers are nearly relaxed, the $P_{\mathrm{I}}$ is mainly the spontaneous polarization. At low bias, any electric field in $\mathrm{GaN}$ is screened by the high electron concentration at the AlGaN/GaN interface. In fact, electrons attracted by the electric field form a $2 \mathrm{D}$ gas at the interface.

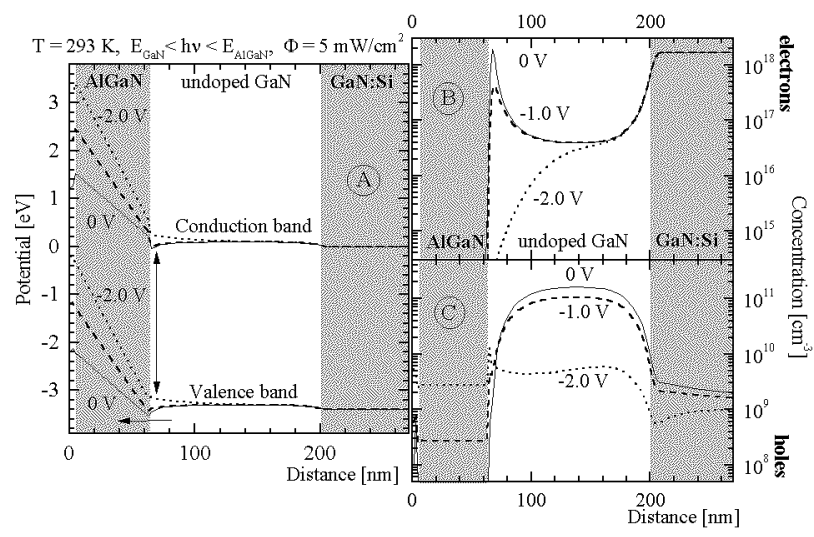

Fig. 3. The structure of the sample with calculated potential (A) and concentration $(\mathrm{B}, \mathrm{C})$ distribution.

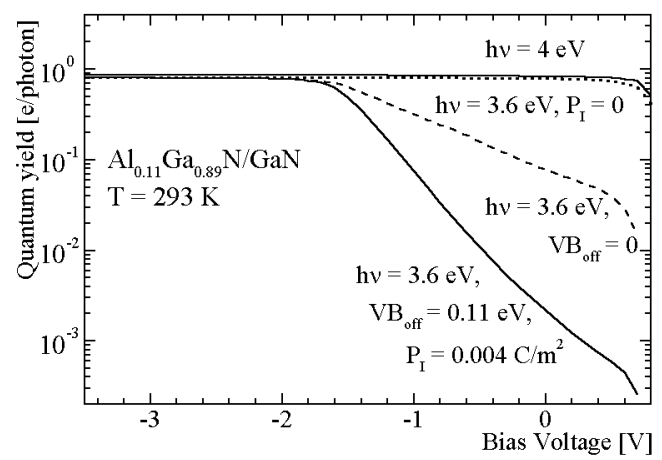

Fig. 4. Quantum yield of AlGaN/GaN structure in different conditions. Solid lines real sample, dashed line - hypothetical sample with valence band offset set to 0 , dotted line - hypothetical sample with polarization on interface set to 0 . Light of $h \nu=3.6 \mathrm{eV}$ excites carriers only in GaN (lower solid line). Light of $h \nu=4.0 \mathrm{eV}$ excites the both layers (higher solid line).

This model allows calculating quantum yield of the structure in different conditions. In the case of high-energy light that excites carriers in both layers 
(GaN and AlGaN), it has been found (see Fig. 4) that the yield practically does not depend upon voltage (under negative bias). In the case of photons that excite carriers only in $\mathrm{GaN}$, the quantum yield strongly depends upon voltage. It changes nearly three orders of magnitude between $U_{\mathrm{B}}=0 \mathrm{~V}$ and $-2 \mathrm{~V}$. This result is in good agreement with experimental data (see Fig. 2).

In order to check what causes the effect, two hypothetical structures have been analyzed: one with valence band offset $\mathrm{VB}_{\text {off }}$ set to 0 and the other, with polarization on interface $P_{\mathrm{I}}$ set to 0 . It has been found (see Fig. 4) that without the polarization, the yield is not sensitive to bias. However, without band offset, the effect is also much weaker. Therefore, both factors, the offset and the polarization are important for bias-dependent spectrum effect. More precise analysis shows that transport of photoexcited holes is crucial to this effect. Photoexcited electrons can flow even at a low electric field. However, holes are stopped at AlGaN/GaN barrier. The accumulated holes cause a fast electron recombination which reduces photocurrent related to the $\mathrm{GaN}(3.4-3.6 \mathrm{eV})$. At a high electric field the barrier becomes triangular and holes can tunnel through it, so the sample is sensitive also to the $3.4-3.6 \mathrm{eV}$ radiation (see experimental results in Fig. 2).

\section{Conclusions}

It has been shown that PC spectrum of a GaN/AlGaN photodetector can depend upon bias voltage $\left(U_{\mathbf{B}}\right)$. Under positive or low negative bias the detector was sensitive mainly to UV radiation absorbed by the AlGaN layer. Application of negative voltage led to the increase in sensitivity connected with photoexcitation of GaN.

The electroreflectance-bias wavelength mapping allowed determination of the electric field in the AlGaN and GaN layers. The field depended on the bias and the polarization on the GaN/AlGaN interface.

Numerical calculations of charge transport show that a barrier formed by valence band offset and the polarization on the interface are crucial for the bias-dependent spectrum effect.

\section{Acknowledgments}

This work was supported in part by Polish Government Project "Blue optoelectronics".

\section{References}

[1] M. Razeghi, A. Rogalski, J. Appl. Phys. 79, 7433 (1996).

[2] D.L. Pulfrey, J.J. Kuek, B.D. Nener, G. Parish, U.K. Mishra, E.J. Tarsa, Physica Status Solidi A 176, 169 (1999). 
[3] S. Nakamura, M. Senoh, S. Nagahama, N. Iwasa, T. Yamada, T. Matsushita, H. Kiyoku, Y. Sugimoto, T. Kozaki, H. Umemoto, M. Sano, K. Chocho, Appl. Phys. Lett. 72, 2014 (1998).

[4] E. Monroy, F. Calle, E. Munoz, F. Omnes, B. Beaumont, P. Gibart, J.A. Munoz, F. Cusso, MRS Internet J. Nitride Semicond. Res. 3, 9 (1998).

[5] O. Ambacher, J. Smart, J.R. Shealy, N.G. Weimann, K. Chu, M. Murphy, W.J. Schaff, L.F. Eastman, R. Dimitrov, L. Wittmer, M. Stutzman, W. Rieger, J. Hilsenbeck, J. Appl. Phys. 85, 3222 (1999).

[6] F. Bernardini, V. Fiorentini, D. Vanderbilt, Phys. Rev. B 56, R10024 (1997).

[7] S. Keller, S. Heikman, L. Shen, I.P. Smorchkova, S.P. DenBaars, U.K. Mishra, Appl. Phys. Lett. 80, 4387 (2002).

[8] T. Tomaszewicz, A. Babinski, D. Suska, J.M. Baranowski, A. Tomaszewicz, Appl. Phys. Lett. 75, 2088 (1999).

[9] D.E. Aspnes, A.A. Studna, Phys. Rev. B 7, 4605 (1973).

[10] A. Drabińska, K.P. Korona, R. Bożek, A. Babiński, J.M. Baranowski, W. Pacuski, R. Stępniewski, T. Tomaszewicz, Phys. Status Solidi B 234, 868 (2002).

[11] K.P. Korona, A. Babiński, J. Kuhl, J.M. Baranowski, R. Leon, Phys. Status Solidi $B$ 227, 605 (2001). 